\author{
H. K. ENGLERT, H. BAUER und H. KUMMER
}

Uber die Fischmehlkrankheit des Schweines . . . . . 195

\author{
W. WUSSOW, I. H. WENIGER, H. R. SCHUMM
}

Die Wirkung einer APF-Beigabe auf Futterverwertung und

Schlachtleistung bel Schweinen . . . . . . . . 223

\title{
HEINZ DREYER
}

Untersuchungen über den Einfluß 3 von Vitamin $B_{1}$, und

APF auf den Eiweißsstoffwechsel........ 240

\begin{abstract}
Das Arohiv für Tierernährung erscheint zweimonatlich in Heften zu 64 Seiten im Format $17,5 \times 25 \mathrm{~cm}$. Der Preis des Heftes betrăgt DM 8,50. 6 Hefte werden zu einem Band vereinigt. Der Besteller muß sich zur Abnahme eines Bandes verpflichten. Die Hefte werden jeweils einzeln bereohnet. Im Jahre erscheint nicht mehr als 1 Band. Bestellungen werden direkt an den AkademieVerlag GmbH., Berlin W 8, MohrenstraBe 39 oder über eine wissenschaftliche Buchhandlung erbeten.
\end{abstract}

Manuskriptsendangen - zugelassen sind die vier KongreBsprachen - sind an den Heraugeber, Herrn Prof. Dr. Ernst Mangold, Berlin N 4, Invalidenstr. 42, zu richten. Mit der Veröffentlichung geht das alleinige Verlagsrecht an das Archiv für Tierernährung über. Daher müssen!Arbeiten, die bereits an anderer Stelle veröffentlicht worden sind, zurückgewiesen werden. Die Verfasser verpflichten sich, Manuskripte, die vom Archiv für Tierernährung angenommen worden sind, nicht an anderer Stelle zu veröffentlichen.

Die Verfasser erhalten von gröBeren wissenschaftlichen Arbeiten 50 Sonderdrucke unentgeltlich. Den Manuskripten beiliegende $\mathrm{Z}$ eichnungen müssen sauber, in zweifacher Größe ausgeführt sein, Wenn sie nicht voll reproduktionsfähig nach den Vorschriften des Normblattes DIN 474 eingereicht werden, ist die Beschriftung nur mit Bleistift einzutragen. Zur Herstellung von Netzätzungen sind nur einwandfreie Photographien brauchbar.

Für alle Literaturzitate sind die Vorschriften des Normblattes DIN 1502 und 1502 Beiblatt I maBgebend. Die Zitate müssen den Verfasser (mit den Anfangsbuchstaben der Vornamen), den vollständigen Titel der Arbeit und die Quelle mit Band, Seitenzahl und Erscheinungsjabr enthalten. Das Literaturverzeichnis soll alphabetisch geordnet sein.

Herausgeber und verantwortlich für den Inhalt: Prof. Dr. med. Dr. phil. Dr. med. vet h. c. Dr. agr. h. c. Ernst Mangold, Berlin N 4, Invalidenstraße 42 (Fernruf 429664 ). Verlag : Akademie-Verlag GmbH., Berlin W 8, MohrenstraBe 39 (Fernruf: 203096 ); Postscheckkonto: 350 21. Bestell- und Verlagsnummer dieses Heftes : 1010/III/4. Das Archiv für Tierernährung erscheint vorläufig jährlich in 1 Band zu 6 Heften. Bezugspreis jo Einzelheft DM 8.50, ausschlieBlich Porto und Verpackung. Satz und Druck: Robert Noske, Borna (Bez. Leipzig). Veröffentlicht unter der Lizenz-Nr. 1213 des Amtes für Literatur und Verlagswesen der Deutschen Demokratischen Republik. Printed in Germany. 